\title{
BMJ Open Exploring the feasibility of establishing a core set of sexual, reproductive, maternal, newborn, child and adolescent health indicators in humanitarian settings: a multimethods, multicountry qualitative study protocol
}

\author{
Loulou Hassan Kobeissi (D) , ${ }^{1}$ Manizha Ashna, ${ }^{2}$ Kassandre Messier, ${ }^{2,3}$ \\ Allisyn C Moran, ${ }^{4}$ Lale Say, ${ }^{5}$ Kathleen Louise Strong, ${ }^{4}$ Angel Foster ${ }^{2}$
}

To cite: Kobeissi LH, Ashna M, Messier $\mathrm{K}$, et al. Exploring the feasibility of establishing a core set of sexual, reproductive, maternal, newborn, child and adolescent health indicators in humanitarian settings: a multimethods, multicountry qualitative study protocol. BMJ Open 2021;11:e041270. doi:10.1136/ bmjopen-2020-041270

- Prepublication history for this paper is available online. To view these files, please visit the journal online (http://dx.doi org/10.1136/bmjopen-2020041270).

LHK and $\mathrm{AF}$ contributed equally.

Received 11 June 2020

Accepted 21 November 2021

Check for updates

(c) Author(s) (or their employer(s)) 2021. Re-use permitted under CC BY-NC. No commercial re-use. See rights and permissions. Published by BMJ.

For numbered affiliations see end of article.

Correspondence to Loulou Hassan Kobeissi; kobeissil@who.int

\section{ABSTRACT}

Introduction In 2019, over 70 million people were forcibly displaced worldwide. Women and girls comprise nearly half of this population and are at heightened risk of negative sexual and reproductive health outcomes. With the collapse of health systems, reduced resources and increased vulnerabilities from displacement, there is a need to strengthen current practices and ensure the delivery of comprehensive sexual, reproductive, maternal, newborn, child and adolescent health (SRMNCAH) services. Recognising the need for consistency in data collection, analysis and use, the WHO developed a list of core SRMNCAH monitoring and evaluation indicators for services and outcomes in humanitarian settings. This research will explore the feasibility of collecting this core set of SRMNCAH indicators in displacement contexts.

Methods and analysis We will undertake a multimethods qualitative study in seven humanitarian settings:

Afghanistan, Albania, Bangladesh, Cameroon, the Democratic Republic of the Congo, Iraq and Jordan. We selected sites that reflect diversity in geographic region, sociocultural characteristics, primary location(s) of displaced persons and nature and phase of the crisis. Our study consists of four components: key informant interviews, facility assessments, observational sessions at select facilities and focus group discussions with frontline healthcare personnel. We will analyse our data using descriptive statistics and for content and themes. We will begin by analysing data from each setting separately and will then combine these data to explore concordant and discordant results, triangulate findings and develop global recommendations.

Ethics and dissemination The University of Ottawa's Research Ethics Board and the Research Project Review Panel (RP 2) of the World Health Organization-Department of Sexual and Reproductive Health as well as local IRBs of PIs' research institutions reviewed and approved this protocol. We intend to disseminate findings through workshops at the WHO country, regional and headquarter levels, as well as through local, national and international
Strengths and limitations of this study

- Our study will obtain field-level perspectives on the feasibility of collecting a core set of sexual, reproductive, maternal, newborn, child and adolescent health (SRMNCAH) indicators for services and outcomes in humanitarian settings.

- Our study includes data collection in multiple countries reflecting a diverse range of humanitarian settings and contexts.

- Our study covers a comprehensive array of SRMNCAH issues and indicators.

- Our study requires extensive fieldwork which may be difficult to conduct given the evolving political, security and COVID-19 contexts.

- Stakeholders may be reluctant to disclose sensitive information related to data collection practices.

conferences, workshops, peer-reviewed publications, and reports.

\section{INTRODUCTION}

Background and context

In 2020, the United Nations High Commissioner for Refugees (UNHCR) estimated that 82.4 million people were forcibly displaced worldwide. ${ }^{1}$ Of this population, the UNHCR classified 25.9 million as refugees, 40.3 million as internally displaced persons and 3.5 million as asylum seekers. ${ }^{1}$ Women and girls of reproductive age comprise roughly half of all displaced people. ${ }^{23}$ In humanitarian contexts, that is, conflict, crisis, refugee and emergency settings, women and girls are at risk of negative sexual and reproductive health (SRH) outcomes. Indeed, evidence from around the world shows that displaced women and girls are at heightened risk of experiencing an 
unintended pregnancy, having an unsafe abortion, dying during pregnancy and delivery, experiencing sexual and gender-based violence and acquiring HIV and other sexually transmitted infections. ${ }^{3-8}$

However, reliable and rigorously collected data on sexual, reproductive, maternal, newborn, child and adolescent health (SRMNCAH) in humanitarian settings are sparse. A 2012-2014 global assessment found significant gaps in information about SRH in refugee and displacement settings, irrespective of region or stage of emergency. ${ }^{9}$ Data gaps were especially pronounced for highly politicised SRH issues, such as abortion. Furthermore, information that is obtained is often variable in content and quality, complicating measurement of the impact of interventions, including life-saving interventions that take place at the outset of an emergency. This lack of data presents challenges for a multitude of stakeholders, including service delivery and implementing organisations, researchers, national governments, international non-governmental organisations (NGOs) and donors thereby impacting the health outcomes of extraordinarily vulnerable populations. Finally, when organisations do collect information about SRMNCAH issues in conflict-affected settings, it is often unclear as to what happens to those data and whether or how they are used to benefit current and future programmatic efforts.

Women and children are vulnerable populations in need of specific essential health services related to pregnancy, newborn health and prevention and treatment of common childhood illnesses. Data on service use and health outcomes for these populations in lower middle income countries typically comes from Demographic and Health Surveys, Multiple Indicator Cluster Surveys or national survey or census efforts. There are no such instruments dedicated to monitoring these populations in humanitarian settings.

Timely and rigorous collection, aggregation and use of SRMNCAH data for services and outcomes evaluation in humanitarian settings is an important component of accountability. Furthermore, this type of data collection could allow governments and implementing agencies to accurately monitor and assess current services and outcomes in humanitarian settings as well as evaluate the impact of programmes and budget allocations. Aligned with target 3.7 of the Sustainable Development Goals to ensure universal access to SRH care services, including for family planning, information and education and the integration of reproductive health into national strategies and programmes, ${ }^{10}$ agencies and organisations require effective systems to collect key information that can track inputs, processes, outcomes and impact. Ideally, this system would be standardised and allow for consistency across all host countries, affected populations and phase of emergency. ${ }^{7}$ Such a system could also promote evidence-based programming and encourage accountability for SRMNCAH service provision among partners.

Since the 1990s, the Inter-Agency Field Manual (IAFM) on Reproductive Health in Humanitarian Settings has provided authoritative guidance on SRH service provision during all phases of complex humanitarian emergencies. ${ }^{11}$ In November 2018, the Inter-Agency Working Group (IAWG) on Reproductive Health in Crises released a new edition of this global resource. ${ }^{12}$ The 2018 IAFM clarified the global standards for assessment, monitoring and evaluation and provided more explicit guidance as to what information should be collected in different phases of an emergency. In 2015, the WHO commissioned a review of data collection tools for maternal and child health in humanitarian settings ${ }^{13}$; in 2018 this review was updated to assess whether recommendations were taken forward and identify new tools. ${ }^{14}$ The findings indicated that there are a wide variety of tools available but not all have been used in the field.

Drawing from the 2018 IAFM and other authoritative guidelines as well as a systematic review of existing indicators,${ }^{15}$ in December 2018, the WHO Departments of Sexual and Reproductive Health and Maternal, Newborn, Child and Adolescent Health and Ageing began the process of developing a common core framework for monitoring SRMNCAH programmes in humanitarian settings. The WHO convened donors, humanitarian partners from UN agencies, representatives from international NGOs and representatives from various WHO regional offices to generate and agree on a core list of SRMNCAH indicators for services and outcomes in humanitarian settings on the basis of existing evidence and programme experiences from the field. In collaboration with global stakeholders, the WHO created a list of core SRMNCAH monitoring and evaluation of services and outcomes indicators for use in humanitarian settings; the Mother and Newborn Information for Tracking Outcomes and Results and Child Health Accountability Tracking technical advisory groups reviewed this list. ${ }^{16}$ The IAWG Safe Abortion Care Sub-Working Group also provided feedback on the abortion-related indicators. The overall aim of this effort is to strengthen current data collection, analysis and use practices.

\section{Study objectives}

Establishing a core set of indicators for routine and universal collection in humanitarian settings represented a first step in this global initiative. However, this candidate set of indicators must be evaluated at the field level to determine feasibility, relevance and acceptability. Building on an ongoing project dedicated to collecting abortion-related data in humanitarian settings, ${ }^{17}$ a multidisciplinary research team from the University of Ottawa collaborated with a team from the WHO to develop a protocol for this assessment. The objectives of our study are to:

1. Assess the feasibility of collecting a core set of SRMNCAH indicators in a diverse array of humanitarian settings.

2. Assess the perceived relevance and usefulness of this set of indicators among a variety of humanitarian stakeholders. 
3. Understand the ability of existing monitoring and evaluation systems to adhere to ethical principles in data collection and safeguard confidentiality and privacy.

4. Identify the data systems and resources required to collect and analyse these core indicators at the field-level.

5. Reach consensus among donor agencies, UN agencies and international NGOs on a minimum set of core SRMNCAH indicators for use in humanitarian settings.

\section{Conceptual framework}

Rogers' diffusion of innovations theory undergirds this project. ${ }^{18}$ We hypothesise that the adoption and diffusion of a core set of SRMNCAH indicators will involve a stage-based progression: awareness of the need for a new intervention; decision to adopt (or reject) the new intervention and continued use of the new intervention. We anticipate that four main factors will influence the feasibility of collecting, analysing and using core SRMNCAH indicators in humanitarian settings.

1. Relative advantage: The degree to which a new intervention is seen as better than the idea, programme or product it replaces.

2. Compatibility: How consistent the new intervention is with the values, experiences and needs of the potential adopters.

3. Complexity: How difficult the new intervention is to understand and/or use?>

4. Observability: The extent to which the new intervention provides tangible results.

Informed by these four factors and a previous study, ${ }^{19}$ we have focused on the following constructs: relevance/ usefulness, feasibility of measurement, systems and resources and ethical issues. Our study design reflects this prioritisation.

\section{METHODS AND ANALYSIS}

Over a 15-month period from September 2019 through December 2020, the University of Ottawa, in partnership with the WHO, will lead the implementation of this study in multiple humanitarian settings.

\section{Study sites}

We intend to conduct this study in seven countries: Afghanistan, Albania, Bangladesh, Cameroon, the Democratic Republic of Congo (DRC), Iraq and Jordan. We purposively selected this set of countries to reflect regional, sociocultural and linguistic diversity (figure 1). Other factors influencing our selection of study sites included:

1. Location of conflict-affected populations: To reflect better various contexts, we intentionally selected countries where refugees and displaced people are predominantly located in camps as well as in urban or non-camp settings. Our sites include locations where displaced populations are both highly accessible and harder to reach.

2. Phase and type of conflict: We purposively selected both protracted displacement settings as well as those that have experienced recent emergencies and/or bursts of acute displacement and service disruption. These sites include settings where populations have been displaced internally as well as across international borders and where displacement has occurred as a result of natural disasters, public health emergencies and conflict/persecution/civil strife.

3. Epidemiological profile: We chose settings that differ by population age structure, mortality rates, causes of death and burden of disease. These settings also reflect significant differences with respect to overall health infrastructure, resources and capacity.

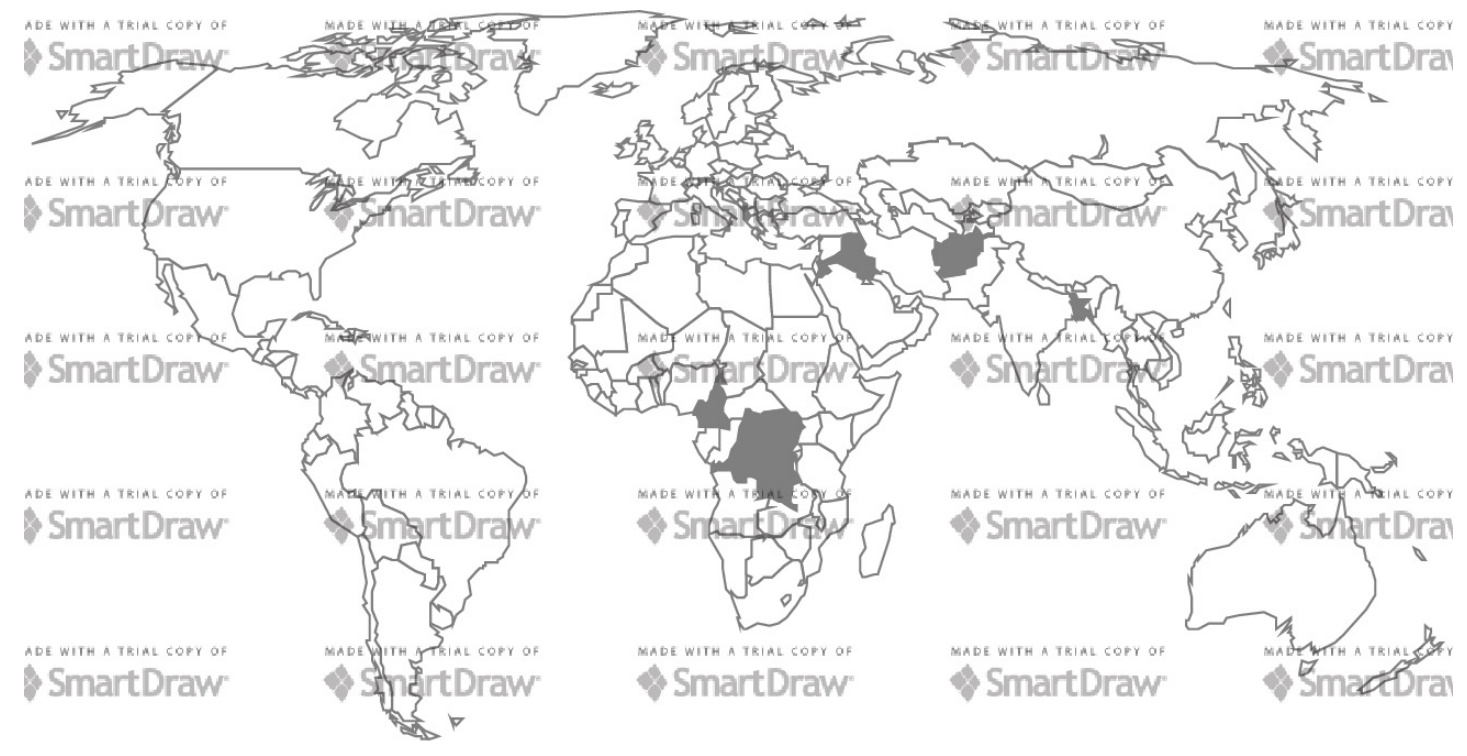

Figure 1 Map of study sites. 
It should be noted that the study was paused in Albania, Cameroon and Iraq due to the COVID-19 pandemic, which currently makes data collection and observations in the field impractical.

\section{Study design}

We modelled our protocol after a number of recent SRH needs and data collection assessments that have taken place in humanitarian settings. ${ }^{17}{ }^{20-22}$ This concurrent multimethods study comprises four core components and will be carried out in each selected country:

1. Key informant interviews (KIIs): We will conduct semistructured interviews with stakeholders who have been purposively selected based on professional position and experience. Through these interviews, we will explore a range of perspectives on data collection and the proposed list of core SRMNCAH indicators.

2. Facility assessments: We will conduct in-person assessments at a purposive selection of primary and tertiary care facilities to understand what data are currently being collected by organisations and identify gaps in data collection. This component of the project will also allow us to create an inventory of available resources and technologies.

3. Observation sessions at select facilities: Non-obtrusive observation sessions will allow us to better understand the logistical, ethical and privacy challenges that organisations currently face when collecting and storing data.
4. Focus group discussions (FGDs): We will invite fieldlevel practitioners at different types of facilities to participate in FGDs to explore attitudes towards data collection, as well as challenges and opportunities for incorporating this new set of SRMNCAH indicators into routine systems.

In collaboration with local partners, we will complete data collection over a 3-6week period in each of the seven settings. The length of fieldwork will depend on the logistics of travelling to different locations within each country.

\section{Sample size and sampling techniques}

We will tailor our samples sizes and sampling techniques to each individual setting. However, we summarise the general parameters of the four study components in table 1 . We have established these targets in order to ensure that we obtain a range of perspectives in a timeframe that is feasible given the multicountry nature of the project.

\section{Data collection}

\section{Predata collection phase}

From the end of 2019 through mid-2020, local project teams in each setting will begin to assemble a list of key agencies involved in the humanitarian response. This process will include engaging with the WHO Country office in each location/region, consulting the SRH Coordinator in each setting, identifying and engaging with the various cluster leads and reaching out to relevant

Table 1 Sample characteristics for each component of the study

\begin{tabular}{|c|c|c|c|}
\hline Study component & Sampling strategy & Participant characteristics & $\begin{array}{l}\text { Estimated number } \\
\text { (per country) }\end{array}$ \\
\hline $\begin{array}{l}\text { Key informant interviews } \\
\text { (KIIs) }\end{array}$ & $\begin{array}{l}\text { Purposive sampling } \\
\text { We will identify well-positioned } \\
\text { individuals based on institutional } \\
\text { affiliation and professional position } \\
\text { through both publicly available } \\
\text { information and professional } \\
\text { networks }\end{array}$ & $\begin{array}{l}\text { Example titles } \\
\text { Executive director, head of } \\
\text { department, reproductive } \\
\text { and maternal health officer, } \\
\text { researcher, epidemiologist, health } \\
\text { information system officer, medical } \\
\text { coordinator, data manager }\end{array}$ & $\begin{array}{l}\text { Total: } 20-30 \\
\text { We anticipate conducting } 5-10 \\
\text { KIIs in each capital city and } \\
5-10 \text { KIIs in each additional } \\
\text { in-country location }\end{array}$ \\
\hline Facility assessments & $\begin{array}{l}\text { Purposive sampling } \\
\text { In consultation with local } \\
\text { stakeholders, we will identify primary } \\
\text { and tertiary facilities that are run by } \\
\text { different organisations in different } \\
\text { displacement locations }\end{array}$ & $\begin{array}{l}\text { Facility types } \\
\text { We will assess two primary care } \\
\text { facilities and one tertiary care } \\
\text { facility in each field-site within the } \\
\text { country }\end{array}$ & Total: 3-9 \\
\hline $\begin{array}{l}\text { Focus group discussions } \\
\text { (FGDs) }\end{array}$ & $\begin{array}{l}\text { Convenience sampling } \\
\text { We will arrange to hold FGDs at a } \\
\text { subset of facilities included in the } \\
\text { facility assessment. We will include } \\
\text { those front-line staff who are working } \\
\text { and available that day }\end{array}$ & $\begin{array}{l}\text { FGD participant characteristics } \\
\text { Front-line staff at each facility } \\
\text { included in facility assessment }\end{array}$ & $\begin{array}{l}\text { Total: } 3-9 \text { FGDs } \\
\text { We anticipate including } 5-10 \\
\text { participants in each group for } \\
\text { a total of } 15-90 \text { participants }\end{array}$ \\
\hline
\end{tabular}


ministries and United Nations agencies. During this preparatory phase, the local team will also identify priority locations within the country to conduct the fieldwork and specific individuals to include in the key informant component of the project. This foundational work will allow us to make final decisions about the length and timing of data collection. We will send key informants the candidate list of indicators approximately 2 weeks before beginning our fieldwork.

\section{Data collection phase}

\section{Key informant interviews}

We aim to obtain a range of perspectives on the proposed set of SRMNCAH indicators by interviewing wellpositioned professionals. Through these interviews, we will explore the utility of collecting SRMNCAH data, challenges related to data collection and ethical practices and safeguards employed. Key informants will also be able to help identify what resources, personnel, funds and infrastructure are needed to collect routine SRMNCAH data. Using an interview guide developed specifically for this study and after obtaining informed consent, a trained researcher will complete the semistructured interview in English and/or a relevant local language; the study team will employ interpreters as needed. We will hold the interviews at a time and location that is convenient for participants; although we expect to conduct all interviews in person, we will offer participants the option to complete the interview over the telephone or Skype. We anticipate interviews will last between 30 and $60 \mathrm{~min}$ and, with permission from the participants, we will audio-record the interviews. The interviewer will take notes during and write analytic memos ${ }^{23}$ immediately after each interaction. The local teams in each country will identify an initial list of key informants; once we begin data collection, we will identify additional informants through early participant referral. In order to obtain a range of perspectives, we expect to conduct 20-30 KIIs in each country.

\section{Facility assessments}

Conducting facility assessments will allow us to document what services are being provided, what information is already being routinely collected and how existing data collection and management practices align with both the proposed set of SRMNCAH indicators and best practices in different types of facilities. Through these assessments, we will also be able to ascertain the source(s) of data, the frequency of data collection and the differences in data collection practices between facilities. As part of the facility assessments, we will record what equipment, software and technology resources are available and used. We have developed a tool for data collection and anticipate that we will work with the clinic manager or a similarly positioned person at each selected facility to complete the assessment. We will conduct these assessments at several primary and tertiary care facilities in each location in each country in order to capture a range of activities and practices and ensure inclusion of facilities run by different organisations. We will identify facilities for this component of the study in consultation with local study teams and input from key informants. The number of facilities will vary by country.

\section{Observation sessions}

Drawing from ethnographic approaches to understanding health systems, practices and adherence to institutional policies, ${ }^{24}$ we will conduct observation sessions at a subset of selected facilities. We will identify sites for inclusion based on our discussions with local stakeholders and scheduling availability/constraints. These observation sessions will allow our study team to understand actual data collection and storage practices. We have designed these observation sessions to be unobtrusive but we may also engage with data managers or data entry personnel while onsite; we will not observe clinical encounters or engage with patients or those seeking services. These sessions will allow us to understand better the degree to which data collection interferes with other priority activities, such as health service delivery. We will take notes during and memo immediately after the observation sessions; there are no specific data collection forms for this component of the study.

\section{Focus group discussions}

In order to understand the experiences and perspectives of front-line staff, we will conduct several FGDs in each country. Through these discussions, we will explore the relevance, feasibility, resources and training and ethical issues associated with current data collection practices as well as collection of the proposed set of core SRMNCAH indicators. We will conduct these FGDs at a subset of the facilities included in the facility assessment component of the project, after discussion with the local team and local stakeholders. Using a facilitation guide developed specifically for this study, a pair of trained researchers will lead each discussion in English and/or a relevant local language, with aid of an interpreter as needed. Consistent with best practices, we aim to include $5-10$ people in each FGD and we will work with local teams to determine the appropriate composition of each FGD and ensure kernels of homogeneity. This will help us identify both social norms and outliers. We anticipate holding at least three FGDs in each country. We expect that the FGDs will take approximately $90 \mathrm{~min}$ and after obtaining informed consent and with permission from the participants, we will audio-record these discussions. We will take notes during, debrief immediately after and analytically memo in the wake of each discussion.

\section{Postdata collection phase}

At the end of this project, we will synthesise the findings and compile country-specific reports outlining recommendations for an intervention to aid data collection, tailored to each humanitarian setting. We will share these recommendations with local stakeholders through presentations and validation workshops in each country. 


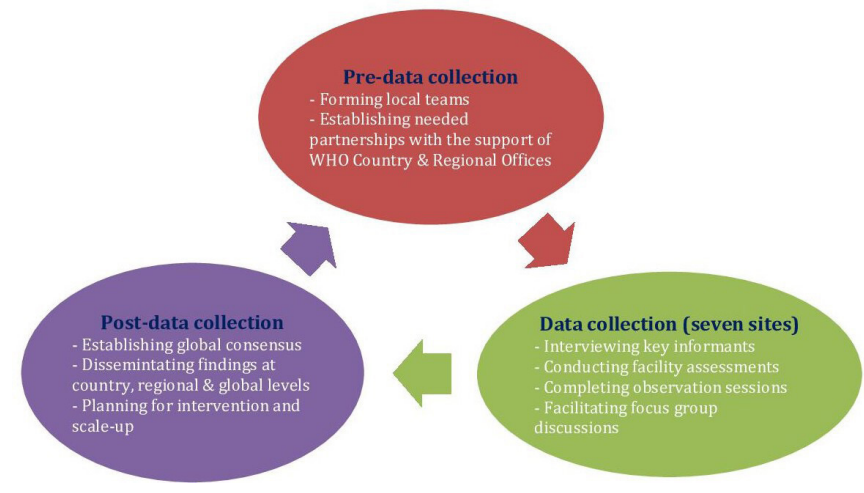

Figure 2 Summary of the data collection phases.

We will also compile the findings from all seven settings into one report in which we will present our overarching findings and recommendations. The WHO will convene a meeting of global-level stakeholders to discuss these findings and recommendations. We anticipate that participants will include representatives from key organisations and agencies, such as donors, UN agencies and international NGOs, working in the humanitarian sector; we will be sure to invite a subset of those who participated in the initial technical consultation that resulted in the candidate set of SRMNCAH indicator. This meeting will serve as a final validation workshop and through this consultation, we aim to achieve consensus on the final set of recommended core SRMNCAH indicators. We provide a summary of these different phases of data collection in figure 2 .

\section{Data analysis}

We will employ a multiphased analytic plan that will begin during data collection. We will analyse the interviews, observation sessions and FGDs for content and themes, using both inductive and deductive techniques. ${ }^{25}{ }^{26} \mathrm{We}$ will analyse the data that we collect through the facility assessments using both descriptive statistics and for content and themes. We will use ATLAS.ti qualitative data management software (Berlin: ATLAS.ti Scientific Software

Development $\mathrm{GmbH}$ ) to organise our data, comprising translated (into English) transcripts, memos and field notes. Our study team will draw from the research questions and our data collection tools to develop an initial codebook; as we review data, we will add and define emergent codes and categories. The Study Coordinator for each setting will serve as the primary coder, the Principal Investigator will audit a subset of coded transcripts and regular team meetings throughout the life of the project will provide us with opportunities to debrief, discuss emergency themes and resolve discrepancies in coding or interpretation through discussion. We will then engage in second and third-level analyses in order to identify key themes and relationships between ideas and attach meaning and significance to the findings. The study team will initially analyse each component of the project from each country separately. We will then bring together the components from each country and integrate the respective findings, paying specific attention to concordant and discordant results. When findings diverge, we will acknowledge and explore factors that might shape this type of variation. In the final analytic phase, we will bring together the synthesised findings from each countryspecific study to identify overarching results. Regular study team meetings will guide our overall interpretation and we will integrate the findings from both local and global validation workshops and consultations into our final recommendations.

\section{PATIENT AND PUBLIC INVOLVEMENT}

Patients and the public were not involved in the design of this study.

\section{ETHICS AND DISSEMINATION}

We received approval from the University of Ottawa's Research Ethics Board to conduct this study in all settings. In addition, the Research Project Review Panel (RP 2) of the World Health Organization-Department of Sexual and Reproductive Health reviewed and approved this protocol. Notably, as part of that approval, the reviewers encouraged our study team to consider the intersection of mental health and SRMNCAH issues when exploring data collection practices and priorities. We subsequently incorporated prompts related to postpartum depression into our interview and FGD guides. We have also received (Afghanistan, Bangladesh, the DRC, Jordan). It should be noted that we have paused the study for Albania, Cameroon and Iraq because of the COVID-19 situation and we are planning to make a decision later depending on the pandemic status. For this reason, approvals from Albania, Cameroon and Iraq to conduct the study from the respective ministries and/or local partners in each setting as well as local ethical approvals from the different local investigators' academic institutions were not obtained and the University of Ottawa's research ethics approval covered: Afghanistan, Cox Bazar in Bangladesh, DRC and Jordan.

We will inform prospective participants that their involvement is voluntary and that any personally identifying information they share with us will be redacted or masked prior to presentation or publication. We will convert hard copies of notes and data collection forms to electronic files and we will store all electronic study materials (including audio-files, memos and transcripts) on password protected computers and secure clouds. Only members of the study team will be able to access these materials.

Our multifaceted dissemination plan includes a suite of activities targeting different audiences. First, we intend to engage in country-specific and setting-specific dissemination through initial validation workshops, end-ofproject presentations and webinars and the release of both country-specific reports and associated executive 
briefs in both English and relevant local languages. We hope that this will help local stakeholders identify ways to strengthen data collection practices in the study sites. Second, we intend to release global recommendations and engage with international, regional and multicountry stakeholders. Our planned consultation with representatives of agencies at the global-level will allow us to develop a feasible and relevant set of recommended SRMNCAH indicators for universal and routine collection in humanitarian settings. Through presentations at international meetings and conferences, release of a multicountry report and publication of peer-reviewed journal articles, we as well aim to share these recommendations with donors, policy-makers, researchers and implementers. Finally, we will work to develop evidence-informed tools to support uptake and implementation of the recommendations. Consistent with our conceptual model, we plan on creating resources and partnering with global bodies, such as the IAWG, to facilitate adoption of the recommendations in a range of humanitarian settings.

\section{ADAPTATIONS IN RESPONSE TO COVID-19}

We developed our study protocol in the fall of 2019, prior to the onset of the global pandemic. We will work closely with local partners in each setting to determine appropriate adaptations to the study protocol to account for travel restrictions, curfews and physical distancing requirements. This will likely include delaying data collection in some settings, conducting KIIs and FGDs through online platforms and conducting virtual facility assessments and observation sessions. As the COVID-19 pandemic has placed increased demands on service providers throughout the world, we will work with our local study teams to minimise the burdens on organisations working in humanitarian settings.

\section{Author affiliations}

${ }^{1}$ Sexual and Reproductive Health and Research, Organisation mondiale de la Sante, Geneve, Switzerland

${ }^{2}$ Faculty of Health Sciences, University of Ottawa, Ottawa, Ontario, Canada

${ }^{3}$ Cambridge Reproductive Health Consultants, Cambridge, Massachusetts, USA

${ }^{4}$ Department of Maternal, Child, Adolescent Health and Aging, World Health

Organization, Geneva, Switzerland

${ }^{5}$ Reproductive Health and Research, World Health Organization, Geneva, Switzerland

\section{Twitter Manizha Ashna @ManizhaAshna}

Contributors LHK and AF conceptualised and designed the study and ACM, KLS and LS reviewed the protocol and provided critical inputs. AF and LHK drafted the initial version of this paper with support from KM and MA. LHK, ACM, KLS and LS reviewed and helped critically revise the paper. AF revised this manuscript after feedback from all other coauthors. All authors approved the submitted version.

Funding This work was funded by the UNDP/UNFPA/UNICEF/WHO/World/Bank Special Programme of Research, Development and Research training in Human Reproduction (HRP) of the Department of Sexual and Reproductive Health and Research at WHO and by WHO through grants from the Department for International Development (UK) and the Ministry of Foreign Affairs of the Netherlands.

Disclaimer The funders had no role in study design, data collection and analysis, decision to publish, or preparation of the manuscript. AMF and her team at the University of Ottawa received funding to develop this protocol from the Canadian Partnership for Women and Children's Health (CanWaCH).
Map disclaimer The inclusion of any map (including the depiction of any boundaries therein), or of any geographic or locational reference, does not imply the expression of any opinion whatsoever on the part of BMJ concerning the legal status of any country, territory, jurisdiction or area or of its authorities. Any such expression remains solely that of the relevant source and is not endorsed by BMJ. Maps are provided without any warranty of any kind, either express or implied.

Competing interests None declared.

Patient and public involvement Patients and/or the public were not involved in the design, or conduct, or reporting or dissemination plans of this research.

Patient consent for publication Not required.

Provenance and peer review Not commissioned; externally peer reviewed.

Open access This is an open access article distributed in accordance with the Creative Commons Attribution Non Commercial (CC BY-NC 4.0) license, which permits others to distribute, remix, adapt, build upon this work non-commercially, and license their derivative works on different terms, provided the original work is properly cited, appropriate credit is given, any changes made indicated, and the use is non-commercial. See: http://creativecommons.org/licenses/by-nc/4.0/.

ORCID iD

Loulou Hassan Kobeissi http://orcid.org/0000-0002-7975-7529

\section{REFERENCES}

1 United Nations High Commissioner for Refugees. Figures at a glance. Available: https://www.unhcr.org/figures-at-a-glance.html [Accessed May 2020].

2 UNHCR-USA. Women. Available: https://www.unhcr.org/en-us/ women.html [Accessed May 2020].

3 Internal displacement monitoring centre. women and girls in internal displacement. Geneva, Switzerland: IDMC, 2020. Available: https:// www.internal-displacement.org/publications/women-and-girls-ininternal-displacement [Accessed May 2020].

4 UNFPA. State of the world population 2015: shelter from the storm. New York, NY: UNFPA, 2015.

5 McGinn T, Casey SE. Why don't humanitarian organizations provide safe abortion services? Confl Health 2016;10:1-7.

6 Austin J, Guy S, Lee-Jones L, et al. Reproductive health: a right for refugees and internally displaced persons. Reprod Health Matters 2008;16:10-21.

7 Askew I, Khosla R, Daniels U, et al. Sexual and reproductive health and rights in emergencies. Bull World Health Organ 2016;94:311.

8 UNAIDS. HIV and refugees, 2007. Available: https://www.unaids.org/ en/resources/presscentre/featurestories/2007/february/20070223 hivandrefugees [Accessed May 2020].

9 Inter-Agency Working Group on Reproductive Health in Crises. Reproductive health in the changing context: Findings from the IAWG on Reproductive Health in Crises' 2012-2014 global evaluation, 2016. Available: https://iawg.net/resources/global-evaluation-2014 [Accessed May 2020].

10 United Nations. Sustainable development goals. Available: https:// sustainabledevelopment.un.org/?menu=1300 [Accessed May 2020].

11 Foster AM, Evans DP, Garcia M, et al. The 2018 Inter-agency field manual on reproductive health in humanitarian settings: revising the global standards. Reprod Health Matters 2017;25:18-24.

12 Inter-Agency Working Group on Reproductive Health in Crises. Interagency field manual on reproductive health in humanitarian settings. New York, NY: IAWG, 2018.

13 Pyone T, Dickinson F, Kerr R, et al. Data collection tools for maternal and child health in humanitarian emergencies: a systematic review. Bull World Health Organ 2015;93:648-58.

14 Constantino JL, Romeiro FD, Diaz T, et al. Data collection tools for maternal and child health in humanitarian emergencies: an updated systematic review. Disaster Med Public Health Prep 2020;14:601-19.

15 Broaddus-Shea ET, Kobeissi L, Ummer O, et al. A systematic review of monitoring and evaluation indicators for sexual and reproductive health in humanitarian settings. Confl Health 2019;13:1-26.

16 WHO. Report for technical consultation for monitoring and evaluation of sexual, reproductive, and maternal, neonatal, child and adolescents health services and outcomes in humanitarian settings. December 11th -13th, 2018.

17 Nara R, Banura A, Foster AM. A Multi-Methods qualitative study of the delivery care experiences of Congolese refugees in Uganda. Matern Child Health J 2020;24:1073-82.

18 Rogers EM. Diffusion of new interventions. New York, NY: Simon and Schuster, 2010. 
19 Saliba D, Solomon D, Rubenstein L, et al. Feasibility of quality indicators for the management of geriatric syndromes in nursing home residents. J Am Med Dir Assoc 2005;6:S50-9.

20 Foster AM, El-Mowafi M, Hammad M, et al. Gender-Based violence and sexual and reproductive health in the South of Jordan: results from a needs assessment and service mapping exercise. Amman, Jordan: UNFPA, Jordan, 2019.

21 Sheehy G, Aung Y, Sietstra C, et al. Near the City but hard to reach: results from a reproductive health needs assessment in periurban Yangon. Cambridge, MA: Cambridge Reproductive Health Consultants, 2015.
22 Hobstetter M, Walsh M, Leigh J, et al. Separated by borders, United in need: an assessment of reproductive health on the Thailand-Burma border. Cambridge, MA: Ibis Reproductive Health, 2012.

23 Birks M, Chapman Y, Francis K. Memoing in qualitative research: probing data and processes. J Res Nurs 2008;13:68-75.

24 Savage J. Ethnography and health care. BMJ 2000;321:1400-2.

25 Deniz N, Lincoln Y. The SAGE Handbook of qualitative research. 3 edn. Thousand Oaks, CA: Sage Publications, 2005.

26 Elo S, Kyngäs $\mathrm{H}$. The qualitative content analysis process. J Adv Nurs 2008;62:107-15. 